\title{
Volcano Activity Relationships for Proton-Coupled Electron Transfer Reactions in Electrocatalysis
}

\author{
Marc T. M. Koper ${ }^{1}$
}

Published online: 24 September 2015

(c) The Author(s) 2015. This article is published with open access at Springerlink.com

\begin{abstract}
This paper studies simple kinetic models for proton-coupled electron transfer reactions, and demonstrates that for reactions in which proton and electron do not transfer simultaneously, $\mathrm{pH}$ dependence of the overall reaction rate is expected. In particular, if the current is evaluated on the reversible hydrogen scale, this may lead to volcano-type activity relations as a function of $\mathrm{pH}$. In case that an acid-base equilibrium is part of the mechanism, the optimal $\mathrm{pH}$ occurs close to the $\mathrm{pK}_{\mathrm{a}}$ of this equilibrium.
\end{abstract}

Keywords Electrocatalysis · Proton-coupled electron transfer $\cdot$ Volcano plot $\cdot \mathrm{pH}$ dependence

\section{Introduction}

Electrocatalysis may be broadly defined as the catalysis of redox reactions [1]. A more specific definition of electrocatalysis highlights the role of "the electrode material on the rate and the mechanism of electrode reactions" [2]. The catalyst's role is to offer alternative pathways for the overall reaction by stabilizing catalytic intermediates through a specific chemical interaction between the intermediates and the catalyst. As a result, the Sabatier principle [3] also applies to electrocatalysis. Volcano activity plots, which depict the activity towards a certain reaction versus

Dedicated to Professor Costas Vayenas on the occasion of 65th birthday and in recognition of his seminal contributions to heterogeneous (electro-)catalysis.

Marc T. M. Koper

m.koper@lic.leidenuniv.nl

1 Leiden Institute of Chemistry, Leiden University, PO Box 9502, 2300 RA Leiden, The Netherlands the energy of stabilization of the key catalytic intermediate (or any other related system parameter), have become highly popular in the heterogeneous electrocatalysis community as a means to organize activity data and to design new catalysts [4-8], though the concept is less widespread in the molecular electrocatalysis community.

However, the electrolyte phase also has a role to play in optimizing the rate of an electrode reaction (or more practically, the output of an electrochemical device). Many electrochemical reactions of practical interest are so-called proton-coupled electron transfer (PCET) reactions, which follow the general reaction equation:

$\mathrm{A}+\mathrm{n} \mathrm{H}^{+}+\mathrm{ne}^{-} \rightleftarrows \mathrm{AH}_{\mathrm{n}}$

Examples include the hydrogen evolution reaction, hydrogen oxidation reaction, oxygen reduction reaction, oxygen evolution reaction, reduction of $\mathrm{CO}_{2}$, oxidation of organic molecules, dinitrogen reduction, ammonia oxidation, and many more [1]. Typically these reactions take place in an aqueous electrolyte, but some of the reactions are also routinely studied in non-aqueous solvents employing suitable proton donors.

Acidity is one of the electrolyte properties that can impact significantly on the rate of an electrode reaction and the performance of an electrochemical device. While this observation is generally acknowledged in the electrochemistry literature, explanations vary and are often very specific to the system under consideration [9]. In this paper, I will show that $\mathrm{pH}$ dependence follows naturally from the general theory of PCET reactions, and may lead to volcano-type activity plots. Thermodynamic arguments for this statement were given in a previous paper [10]. Here, I will consider simple kinetic models, and I will emphasize the idea to view the electrochemical system as a whole, that is: to include the fact that the $\mathrm{pH}$ dependence of the 
reaction under consideration must be compared to the $\mathrm{pH}$ dependence of the second (counter or reference) electrode to understand the "final" impact of $\mathrm{pH}$ on device performance. While this is somehow natural to people working in the field of fuel cells and water electrolysis, this idea is not generally included in the PCET theory. This difference in viewpoint, though conceptually rather trivial, is often at the basis of the confusion that arises when the heterogeneous electrocatalysis community and the PCET community argue about $\mathrm{pH}$ dependence.

\section{Model: Results and Discussion}

The molecular-level theory of PCET reactions is well developed; excellent reviews are available on various experimental and theoretical aspects [11-14]. In essence, PCET theory models a reaction scheme such as shown in the upper panel of Fig. 1, i.e. reaction 1 with $n=1$, and considers (or computes) a potential energy surface for such a reaction as shown in the lower panel. One of the key questions that PCET theory tries to resolve is whether
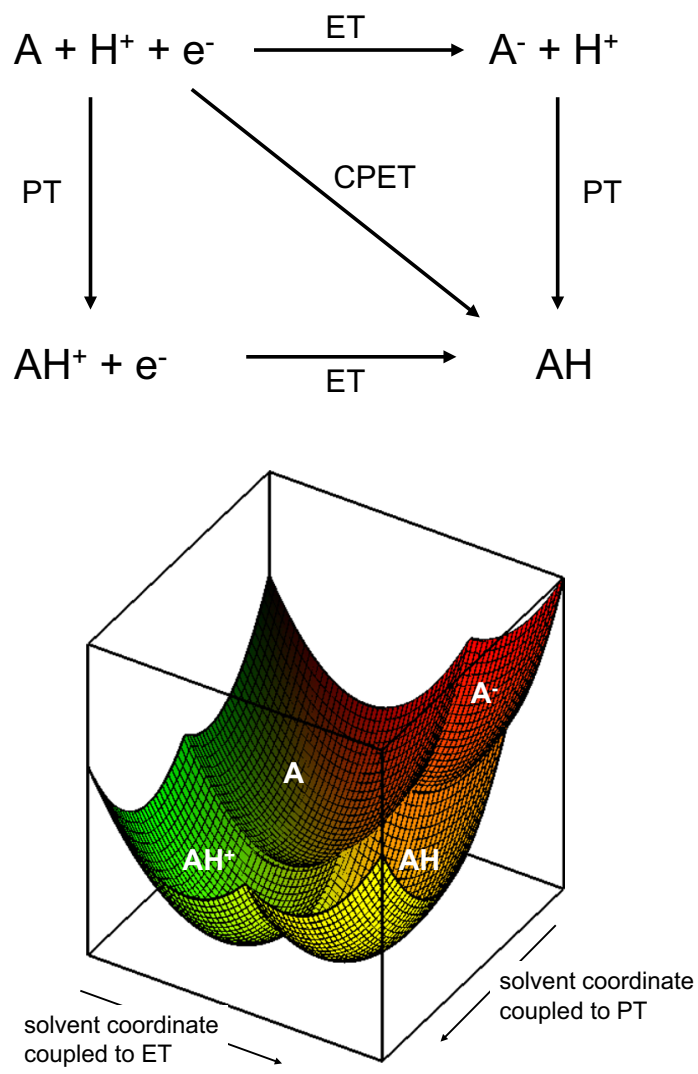

Fig. 1 Upper panel square scheme of PCET, following ET, proton transfer (PT) and concerted proton-electron transfer (CPET) steps. Lower panel potential energy surface representation of the PCET as a function of the generalized solvent coordinates coupled to ET and PT proton and electron transfer take place simultaneously, a phenomenon referred to as concerted proton-coupled electron transfer (CPET), or whether they take place successively, in sequential pathways with first electron and next proton transfer or vice versa. These are the "diagonal" and "off-diagonal" pathways, respectively, shown in the scheme in Fig. 1. The choice of pathway mainly depends of the free energies of the "off-diagonal" states $\mathrm{HA}^{+}$and $\mathrm{A}^{-}$, and on the activation energies associated with electron transfer (ET), proton transfer (PT), and CPET steps.

Detailed expressions exist for the rates and the energies of activation of the various pathways shown in Fig. 1 [10, 13, 15, 16]. In general, CPET takes place if the free energies of the off-diagonal states $\mathrm{AH}^{+}$and $\mathrm{A}^{-}$are high. If the free energies of either $\mathrm{AH}^{+}$or $\mathrm{A}^{-}$are comparable to $\mathrm{AH}$, the activation energies for the sequential ET and PT steps are generally lower than that for the concerted step, and the reaction typically follows a sequential pathway ("decoupled proton-electron transfer") [10].

In the remainder of this section, I will consider simple kinetic models for two typical situations of PCET: the situation in which proton transfer (or deprotonation) precedes ET, and the situation in which ET (or reduction) precedes proton transfer. All equations to be derived below are steady-state expressions in which the eventual effect of slow diffusion of reactants is not accounted for.

For the first example, consider the following mechanism for the oxidation of a molecule HA:

$\mathrm{HA} \rightleftarrows \mathrm{H}^{+}+\mathrm{A}^{-}$

$\mathrm{A}^{-} \rightarrow \mathrm{A}+\mathrm{e}^{-}$

Such a mechanism (albeit more detailed) has recently been proposed for the oxidation of formic acid [17] as well as for the oxidation of various alcohols [18]. If in this mechanism reaction 3 is irreversible, one obtains the following expression for the current density in the steady-state ("ss") approximation:

$$
\begin{aligned}
j & =F k_{2}\left[A^{-}\right]^{S S}=\frac{F k_{2}[H A]^{0}}{1+\frac{k_{-1}}{k_{1}}\left[H^{+}\right]+\frac{k_{2}}{k_{1}}} \\
& =\frac{F k_{2}^{0}[H A]^{0} \exp \left(\frac{\alpha F\left(E_{S H E}-E_{A / A^{-}}^{0}\right)}{R T}\right)}{1+10^{p K_{a}-p H}+\frac{k_{2}^{0}}{k_{1}} \exp \left(\frac{\alpha F\left(E_{S H E}-E_{A / A^{-}}^{0}\right)}{R T}\right)}
\end{aligned}
$$

where $\mathrm{pK}_{\mathrm{a}}$ is the acidity constant of $\mathrm{HA}$, and where the rate of reaction 3 has been assumed to follow the ButlerVolmer law. In this equation, $k_{1}$ and $k_{-1}$ are the rate constants corresponding to reaction $2,[H A]^{0}$ is the initial nominal concentration of HA, $k_{2}^{0}$ is the standard heterogeneous rate constant for reaction $3, \alpha$ is the so-called Butler-Volmer transfer coefficient (often equal to 0.5 though in reality it may be mildly potential dependent [1] ), 


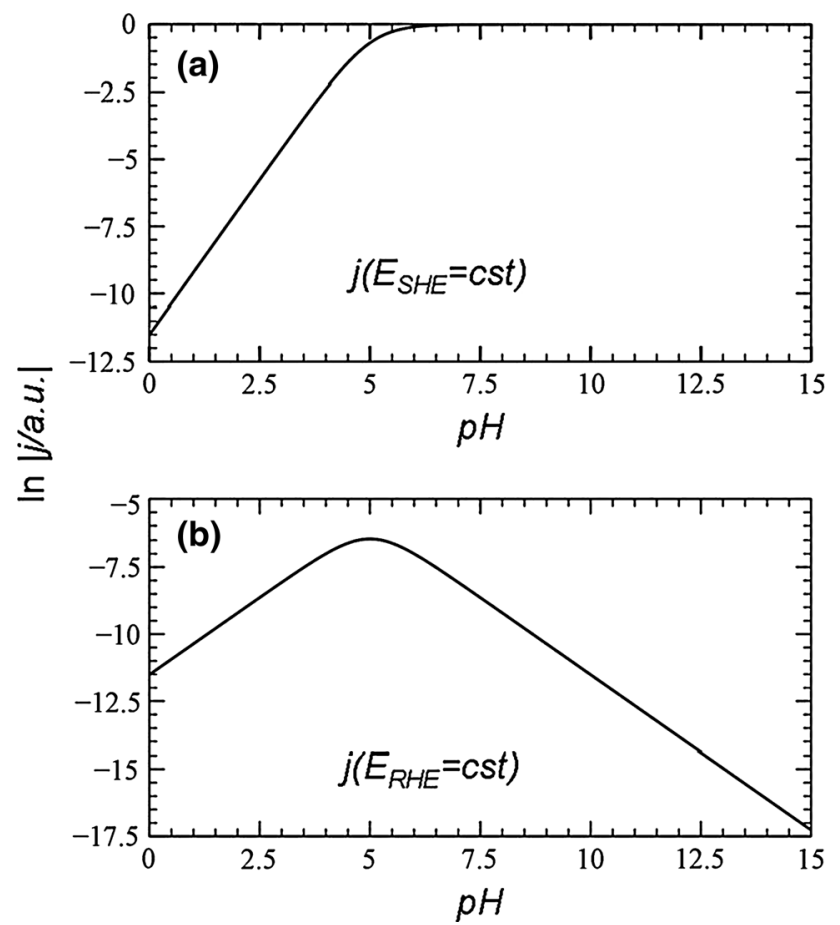

Fig. 2 a Typical plot of Eq. $3 ; F k_{2}[H A]^{0}=1, \mathrm{pK}_{\mathrm{a}}=5, k_{2} / k_{1}=$ $0.01, E_{S H E}-E_{A / A^{-}}^{0}=0$. b Typical plot of Eq. $5 ; E_{R H E}-E_{A / A^{-}}^{0}=0$; $\alpha=0.5$, other parameter choices as in $\mathbf{a}$

$E_{A / A^{-}}^{0}$ is the standard equilibrium potential for reaction 3, and $F, R$, and $T$ have their usual meaning. Equation 4 specifies explicitly that the potential is referred to the standard hydrogen electrode, for reasons that will become apparent below. Figure 2a shows the current (evaluated at an arbitrary constant value of $E_{S H E}$ ) as a function of $\mathrm{pH}$, as predicted by Eq. 4, showing an initially low rate at low $\mathrm{pH}$ since no $\mathrm{A}^{-}$is available, and leveling off to a constant high rate at high $\mathrm{pH}$ at which all $\mathrm{HA}$ in solution has been converted into $\mathrm{A}^{-}$. This is the standard S-shaped curve for $\mathrm{pH}$ dependent reactivity seen in the molecular electrocatalysis literature [19].

In the heterogeneous electrocatalysis literature, it is customary to take into account the $\mathrm{pH}$ dependence of the reference electrode, since in a real device, the second electrode will also have a $\mathrm{pH}$ dependence so that overall no protons are produced or consumed in the cell (i.e. the cell operates at constant $\mathrm{pH}$ ). Ideally this second electrode is entirely reversible involving concerted proton-electron transfer, so it is often the hydrogen electrode in the same solution with the same $\mathrm{pH}$. The electrode potential vs the reversible hydrogen electrode RHE is related to the electrode potential versus the SHE by:

$$
\begin{aligned}
E_{S H E} & =E_{R H E}-0.059 p H=E_{R H E}-\frac{R T \ln 10}{F} p H \\
& =E_{R H E}+\frac{R T}{F} \ln \left[H^{+}\right]
\end{aligned}
$$

Using this RHE scale as the "relevant" scale, the equation for the current density becomes:

$$
j=\frac{F k_{2}^{0}[H A]^{0}\left(10^{-p H}\right)^{\alpha} \exp \left(\frac{\alpha F\left(E_{R H E}-E_{A / A^{-}}^{0}\right)}{R T}\right)}{1+10^{p K_{a}-p H}+\frac{k_{2}^{0}}{k_{1}}\left(10^{-p H}\right)^{\alpha} \exp \left(\frac{\alpha F\left(E_{R H E}-E_{A / A^{-}}^{0}\right)}{R T}\right)}
$$

This equation predicts a volcano-type curve for the current (now evaluated at an arbitrary constant value of $E_{R H E}$ ), as illustrated in Fig. 2b, with a maximum current for:

$p H=p K_{a}+\log \frac{\alpha}{1-\alpha}$

Since typically $\alpha \approx 0.5$, this equation reduces to $\mathrm{pH}=\mathrm{pK}_{\mathrm{a}}$. This implies that in a device with the above "slow" oxidation reaction at the anode, and a fast reversible $\mathrm{H}^{+} / \mathrm{e}^{-}$CPET reaction at the other electrode, the current output maximizes at $\mathrm{pH}=\mathrm{pK}_{\mathrm{a}}$. The reason for the increasingly lower reaction rate for $\mathrm{pH}>\mathrm{pK}_{\mathrm{a}}$ is the fact that at this $\mathrm{pH}$ the concentration of $\mathrm{A}^{-}$has saturated (and it is therefore no longer $\mathrm{pH}$ dependent) but the rate of ET of reaction 3 is evaluated at increasingly higher overpotential because of the $\mathrm{pH}$ dependent reference potential. This $\mathrm{pH}$ dependent volcano-type activity plot has been observed experimentally for the electrocatalytic oxidation of formic acid on platinum and gold electrodes [17, 20], but also for the homogeneously catalyzed oxidation of formic acid by a molecular iridium-ruthenium complex [21].

It is important to emphasize that there is no fundamental difference between Fig. 2a and b; they are different ways of plotting the same result. Using the RHE reference, one "corrects" for the expected $\mathrm{pH}$ dependence of concerted proton-electron transfer, i.e. the $60 \mathrm{mV} / \mathrm{pH}$ shift observed on the SHE scale. In the heterogeneous electrocatalysis community, CPET is often considered the norm and therefore it is logical to consider $\mathrm{pH}$ dependence on the RHE scale, since the RHE scale incorporates this intrinsic $\mathrm{pH}$ dependence of CPET processes. A half cell reaction showing $\mathrm{pH}$ dependence on the RHE scale hence implies the existence of decoupled proton-electron transfer pathways. On the other hand, in the molecular electrocatalysis literature, CPET pathways are considered more exceptional, and decoupled pathways are the norm. Hence, there is no tradition to employ a $\mathrm{pH}$ corrected reference electrode such as the RHE. In addition, in non-aqueous solvents, as often employed in molecular electrocatalyis studies, there exists no obvious practical alternative for the RHE in aqueous electrolytes.

Next, let us consider the reverse situation of Eqs. 2 and 3 , where ET takes place first, and proton transfer is a next irreversible step:

$$
\begin{aligned}
& \mathrm{A}+\mathrm{e}^{-} \rightleftarrows \mathrm{A}^{-} \\
& \mathrm{H}^{+}+\mathrm{A}^{-} \rightarrow \mathrm{HA}
\end{aligned}
$$


An example of such a reaction mechanism would be the reduction of oxygen on a weakly interacting electrode material such as gold, mercury or graphite in aqueous media, where the first intermediate has been suggested to be $\mathrm{O}_{2}{ }^{-}$superoxide anion [10]. In the steady-state approximation for $\mathrm{A}^{-}$, we have:

$\left[A^{-}\right]^{s s}=\left(\frac{k_{-2}}{k_{2}[A]}+\frac{k_{1}\left[H^{+}\right]}{k_{2}[A]}\right)^{-1}$

Because reaction 2 is now assumed irreversible, the measured steady-state current is equal to the rate of formation of HA, since this is the reaction that causes the ET reaction 3 to be out-of-equilibrium and hence yield an effective reduction current. Therefore:

$j=-\frac{F k_{1} 10^{-p H}}{\frac{k_{-2}^{0} \exp \left(\frac{F\left(E_{S H E}-E_{2}^{0}\right)}{R T}\right)}{k_{2}^{0}[A]}+\frac{k_{1} 10^{-p H} \exp \left(\frac{\alpha F\left(E_{S H E}-E_{2}^{0}\right.}{R T}\right)}{k_{2}^{0}[A]}}$

or

$$
j=-\frac{F k_{1} 10^{-p H}}{\frac{k_{-2}^{0} 10^{-p H} \exp \left(\frac{F\left(E_{R H E}-E_{2}^{0}\right)}{R T}\right)}{k_{2}^{0}[A]}+\frac{k_{1}\left(10^{-p H}\right)^{1+\alpha} \exp \left(\frac{\alpha F\left(E_{R H E}-E_{2}^{0}\right.}{R T}\right)}{k_{2}^{0}[A]}}
$$

Figure 3 plots these curves as a function of $\mathrm{pH}$ for representative values of the various parameters. On the SHE scale, the reaction slows down with increasing $\mathrm{pH}$, because the driving force for ET remains the same on this potential scale but the rate of the subsequent PT slows down with higher $\mathrm{pH}$. However, this is probably not realistic, at least not in aqueous media. In the absence of sufficient protons, it is likely that reaction 2 will take place through a reaction with water.

$\mathrm{H}_{2} \mathrm{O}+\mathrm{A}^{-} \rightarrow \mathrm{HA}+\mathrm{OH}^{-}$

Depending on the rate of reaction $2 \mathrm{~b}$ (with corresponding rate constant $k_{l b}$ ), i.e. in which water is the proton donor, the rate at high $\mathrm{pH}$ will settle onto a constant value, as illustrated by the dotted line. On the RHE scale, the reaction rate increases with increasing $\mathrm{pH}$, as the ET reaction is probed at increasingly lower overpotential. The saturation is again due to the lower availability of protons, which exactly counteracts this effect, at least in the model. In reality, water will likely act as proton donor at such high $\mathrm{pH}$, and no such saturation is expected, as illustrated by the dotted line. The observation that the rate of oxygen reduction increases with $\mathrm{pH}$ agrees well with experiments on gold electrodes: the oxygen reduction proceeds much closer to the equilibrium potential of the overall oxygen reduction to water (i.e. $1.23 \mathrm{~V}$ vs RHE) in alkaline media than in an acidic media [22]. The fact that the mechanism for the oxygen reduction reaction on gold proceeds through a superoxide anion intermediate is therefore the reason why
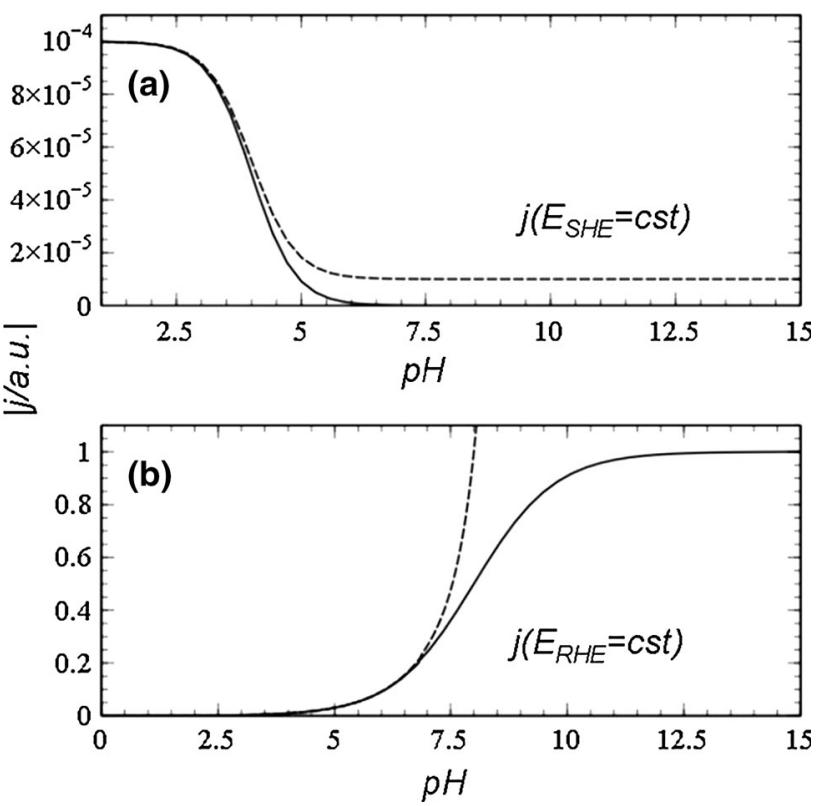

Fig. 3 a Typical plot of Eq. 8; $\quad F k_{1}=1, \quad k_{-2}^{0} / k_{2}^{O}[A]=1, \quad k_{1} /$ $k_{2}^{O}[A]=10^{4}$. Solid line $k_{1 b}=0$; dashed line $k_{1 b}=10^{-5}$. b Typical plot of Eq. $9 ; \alpha=0.5$, other parameter choices as in a. $E-E^{0}=0$ in both figures

gold is such a good electrode material for oxygen reduction in alkaline media [10, 23].

In some mechanisms, we may need to consider that $\mathrm{A}^{-}$ is an intermediate with a maximum concentration, e.g. because it is bound to the catalyst. In such a case, our model becomes similar to classical models suggested many years ago by Laviron [24]. Experimental examples of this situation would include the reduction of $\mathrm{CO}_{2}$ to a catalystbound $\mathrm{CO}_{2}{ }^{-}$intermediate, a mechanism regularly suggested for $\mathrm{CO}_{2}$ reduction on metal complexes [25], or the reduction of $\mathrm{CO}$ on $\mathrm{Cu}(100)$ electrodes, which has been suggested to proceed through the formation of an adsorbed $(\mathrm{CO})_{2}^{-}$intermediate $[26,27]$. We now explicitly take into account the rate of reaction $2 \mathrm{~b}, k_{1 b}$, for reasons that will become apparent below. For this model, Eq. 8 changes to:

$\left[A^{-}\right]^{s s}=\left(1+\frac{k_{-2}}{k_{2}[A]}+\frac{k_{1}\left[H^{+}\right]+k_{1 b}}{k_{2}[A]}\right)^{-1}$

Equations 9 and 10 become:

$j=-\frac{F\left[k_{1} 10^{-p H}+k_{1 b}\right]}{1+\frac{k_{-2}^{0} \exp \left(\frac{F\left(E_{S H E}-E_{2}^{0}\right)}{R T}\right)}{k_{2}^{0}[A]}+\frac{\left(k_{1} 10^{-p H}+k_{1 b}\right) \exp \left(\frac{\alpha F\left(E_{S H E}-E_{2}^{0}\right.}{R T}\right)}{k_{2}^{0}[A]}}$

$j=-\frac{F\left[k_{1} 10^{-p H}+k_{1 b}\right]}{1+\frac{k_{-2}^{0} 10^{-p H} \exp \left(\frac{F\left(E_{R H E}-E_{2}^{0}\right)}{R T}\right)}{k_{2}^{0}[A]}+\frac{\left[k_{1} 10^{-p H}+k_{1 b}\right]\left(10^{-p H}\right)^{\alpha} \exp \left(\frac{\alpha F\left(E_{R H E}-E_{2}^{0}\right.}{R T}\right)}{k_{2}^{0}[A]}}$ 


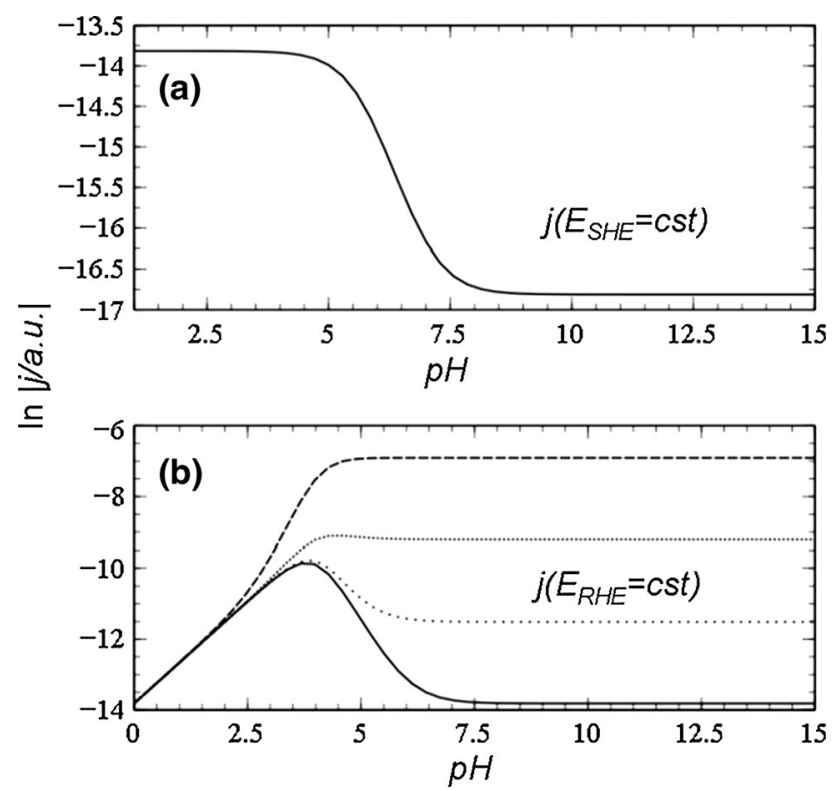

Fig. 4 a Typical plot of Eq. 11; $F k_{1}=1, \quad F k_{I b}=10^{-7}, \quad k_{-2} l$ $k_{2}[A]=1, k_{l} / k_{2}[A]=10^{6}$. b Typical plot of Eq. $12 ; \alpha=0.5$, solid line $F k_{1 b}=10^{-6}$, other lines $F k_{1 b}=10^{-5}, 10^{-4}, 10^{-3}$ (from bottom to top); other parameter choices as in a. $E-E^{O}=0$ in both figures

Figure 4 plots the $j$ versus $\mathrm{pH}$ curves predicted by Eqs. 12 and 13; the latter curve for various values of $k_{l b}$. The most interesting observation is that, depending on the (relative) value of $k_{1 b}$, the current vs. pH plot using the RHE scale develops a maximum. For $k_{l b} \rightarrow 0$, this happens for:

$p H=\frac{1}{1+\alpha} \log \left(\frac{\alpha k_{1} \exp \left(\frac{\alpha F\left(E_{R H E}-E_{2}^{0}\right.}{R T}\right)}{k_{2}^{0}[A]}\right)$

Since the acid-base reaction is considered out-of-equilibrium, its $\mathrm{pK}_{\mathrm{a}}$ plays no role in the maximum activity, at least not in this simple kinetic model. The $\mathrm{pH}$ of maximum activity now depends on the relative rates of formation and protonation of the $\mathrm{A}^{-}$intermediate (Eq. 14). The reason for the maximum lies in the fact in this version of the model, there is saturation in the concentration of the intermediate. When this saturation is reached, higher values of the $\mathrm{pH}$ slow down the overall reaction rate on the RHE scale. Whether the maximum actually develops depends on the rate of reaction $2 b$, as can be seen from Fig. $4 b$. If this reaction is fast, this alternative protonation pathway will dominate and the rate will become constant at high $\mathrm{pH}$ due to the $\mathrm{pH}$ independent rate of this reaction.

\section{Conclusion}

This paper has considered $\mathrm{pH}$ dependent reactivity maps for electrochemical PCET reactions in which sequential proton-electron transfer takes place. The simple kinetic modeling theory presented here builds on previous Hamiltonian modeling [28] and thermodynamic considerations [10]. The modeling confirms the role of $\mathrm{pH}$ in optimizing the reactivity of electrocatalytic reactions, leading to volcano-type activity relations if acid-base equilibria are involved in the reaction mechanism. The importance of considering the potential reference scale (i.e. SHE and RHE) has also been illustrated, which should be helpful in avoiding future confusion about the interpretation of " $\mathrm{pH}$ dependence" of PCET reactions.

Acknowledgments I would like to acknowledge the Université de Paris-Denis Diderot for a visiting professorship and Professors Cyrille Costentin, Marc Robert, and Jean-Michel Savéant at the Laboratoire d'Electrochimie Moleculaire of the Université de Paris-Denis Diderot for very helpful discussions.

Open Access This article is distributed under the terms of the Creative Commons Attribution 4.0 International License (http://crea tivecommons.org/licenses/by/4.0/), which permits unrestricted use, distribution, and reproduction in any medium, provided you give appropriate credit to the original author(s) and the source, provide a link to the Creative Commons license, and indicate if changes were made.

\section{References}

1. Koper MTM (2013) Catalysis of redox reactions. In: Yam VWW, Reedijk J, Poeppelmeier K (eds) Comprehensive inorganic chemistry II, vol 8. Elsevier, Amsterdam, pp 459-474

2. Trasatti S (1995) Int J Hydrogen Energy 20:835

3. Sabatier P (1911) Ber Deutsch Gem Ges 44:1984

4. Trasatti S (1972) J Electroanal Chem 39:163

5. Petrii OA, Tsirlina GA (1994) Electrochim Acta 39:1739

6. Nørskov JK, Bligaard T, Logadottir A, Kitchin JR, Chen JG, Pandelov S, Stimming U (2003) J Electrochem Soc 152:J23

7. Appleby AJ, Zagal JH (2011) J Solid State Electrochem 15:1811

8. Koper MTM (2011) J Electroanal Chem 660:254

9. Tarasevich MR, Korchagin OV (2013) Russ J Electrochem 49:600

10. Koper MTM (2013) Chem Sci 4:2710

11. Cukier RI, Nocera DG (1998) Annu Rev Phys Chem 49:337

12. Mayer JM (2004) Annu Rev Phys Chem 55:363

13. Hmmes-Schiffer S, Stuchebrukhov AA (2010) Chem Rev 110:6939

14. Savéant JM (2014) Annu Rev Anal Chem 7:537

15. Costentin C, Robert M, Savéant JM (2006) J Electroanal Chem 588:197

16. Grimminger J, Bartenschlager S, Schmickler W (2006) Chem Phys Lett $416: 316$

17. Joo J, Uchida T, Cuesta A, Koper MTM, Osawa M (2013) J Am Chem Soc 135:9991

18. Kwon Y, Lai SCS, Rodriguez P, Koper MTM (2011) J Am Chem Soc 133:6914

19. Bonin J, Costentin C, Robert M, Routier M, Savéant JM (2013) J Am Chem Soc 135:14359

20. Brimaud S, Solla-Gullon J, Weber I, Feliu JM, Behm RJ (2014) ChemElectroChem 1:1075

21. Fukuzumi S, Kobayashi T, Suenobu T (2010) J Am Chem Soc 132:1496 
22. Mei D, He ZD, Zheng YL, Jiang DC, Chen YX (2014) Phys Chem Chem Phys 16:13762

23. Quaino P, Luque N, Nazmutdinov RR, Santos E, Schmickler W (2012) Angew Chem Int Ed 51:12997

24. Laviron E (1974) J Electroanal Chem 52:355

25. Savéant JM (2008) Chem Rev 108:2348
26. Schouten KJP, Qin Z, Perez Gallent E, Koper MTM (2012) J Am Chem Soc 134:9864

27. Calle-Vallejo F, Koper MTM (2013) Angew Chem Int Ed 52:7282

28. Koper MTM (2013) Phys Chem Chem Phys 15:1399 